\title{
Prenatal Diagnosis of Aorta-Porto-Umbilical Vein Fistulas with Left-Sided Inferior Vena Cava
}

\author{
Jiawei Zhou, $\mathrm{PhD}^{1} \quad$ Qichang Zhou, $\mathrm{PhD}^{1} \quad$ Qianghai Peng, $\mathrm{PhD}^{1} \quad$ Shi Zeng, $\mathrm{PhD}^{1}$ \\ 1 Department of Ultrasonography, The Second Xiangya Hospital, \\ Central South University, Changsha, Hunan, People's Republic of \\ China \\ Address for correspondence Shi Zeng, PhD, Department of \\ Ultrasound, The Second Xiangya Hospital, Central South University, \\ 139 Renmin Road (M), Changsha 410011, Hunan, People's Republic of \\ China (e-mail: doctorzshi@163.com).
}

Am J Perinatol Rep 2016;6:e59-e61.
Abstract Keywords
- fetal
- fistulas
- left-sided inferior vena cava

Congenital arteriovenous fistulas involved with the abdominal aorta are very rare. Leftsided subrenal inferior vena cava (IVC) with normal connection to the heart is also rare and has not been reported prenatally. In this article, we described a fetus with aortaporto-umbilical vein fistulas combined with a left-sided IVC.

\section{Case Study}

A 32-year-old, gravida 2 para 1 woman was referred to our center for fetal echocardiography at $30^{+4}$ weeks of gestation following routine ultrasound screening because of cardiomegaly. Family history was negative for congenital heart malformations, and there was no history of medication use during pregnancy. The cardiothoracic ratio was 0.49 , and there was no sign of hydrops. Echocardiography showed normal segmental anatomy and a perimembranous ventricular septal defect. On detailed examination, it was noted that the dilated intra-abdominal part of umbilical vein coursed into the liver like a helix. Doppler imaging demonstrated a $28 \times 24 \mathrm{~mm}$ vascular structure in the middle part of the liver and revealed a "saw-toothed" arterial flow pattern with an extremely high-peak velocity $(240 \mathrm{~cm} / \mathrm{s})$ in the lesion (-Fig. 1). Subtle examination of the lesion on sagittal and transverse views using color and power Doppler revealed that the following vessels were in continuity with the lesion: an arterial vessel originating directly from the abdominal aorta, portal veins, and a dilated umbilical vein (-Fig. 2). Moreover, the subrenal inferior vena cava (IVC) ran upward along the left side of the aorta and then anteriorly crossed the aorta at the level of the kidney to join the normal IVC on the right (-Fig. 3). All three hepatic arteries and ductus venosus were visible and coursed normally. Cordocentesis yielded a normal karyotype (46, XY). The pregnancy was uneventful and showed no sign of hydrops, and spontaneous vaginal delivery occurred at 37 weeks' of gestation. An extended right hepatectomy was successfully performed at the age of 3 months. There were no complications, and sonography showed no obvious residual lesions upon follow-up after 3 months.

\section{Discussion}

Congenital arteriovenous shunts in the liver of fetuses are very rare, especially when involved with the abdominal aorta. ${ }^{1}$ Portal hypertension and/or congestive heart failure are the main presentations in postnatal life. Although the progress of the arteriovenous malformation in the fetus depends on the size of the lesion, the related arteries and the associated anomalies, most published isolated cases showed good outcomes including spontaneous closure ${ }^{2}$ and successful surgery. ${ }^{3}$

This anomaly has mostly presented alone, with a few cases been associated with other congenital defects, such as duodenal atresia and trisomy $21 .^{1}$ To the best of our knowledge, the combination of defects described in this case, namely, aorta-porto-umbilical vein fistulas with a left-sided IVC, has not been previously reported. Left-sided IVC is a rare form of IVC malformation. A search of the received

July 27, 2015

accepted after revision

September 9, 2015

published online

October 28, 2015
DOI http://dx.doi.org/

10.1055/s-0035-1566244. ISSN 2157-6998.
Copyright $\odot 2016$ by Thieme Medical Publishers, Inc., 333 Seventh Avenue, New York, NY 10001, USA. Tel: +1(212) 584-4662.
License terms

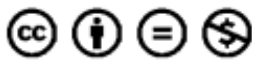




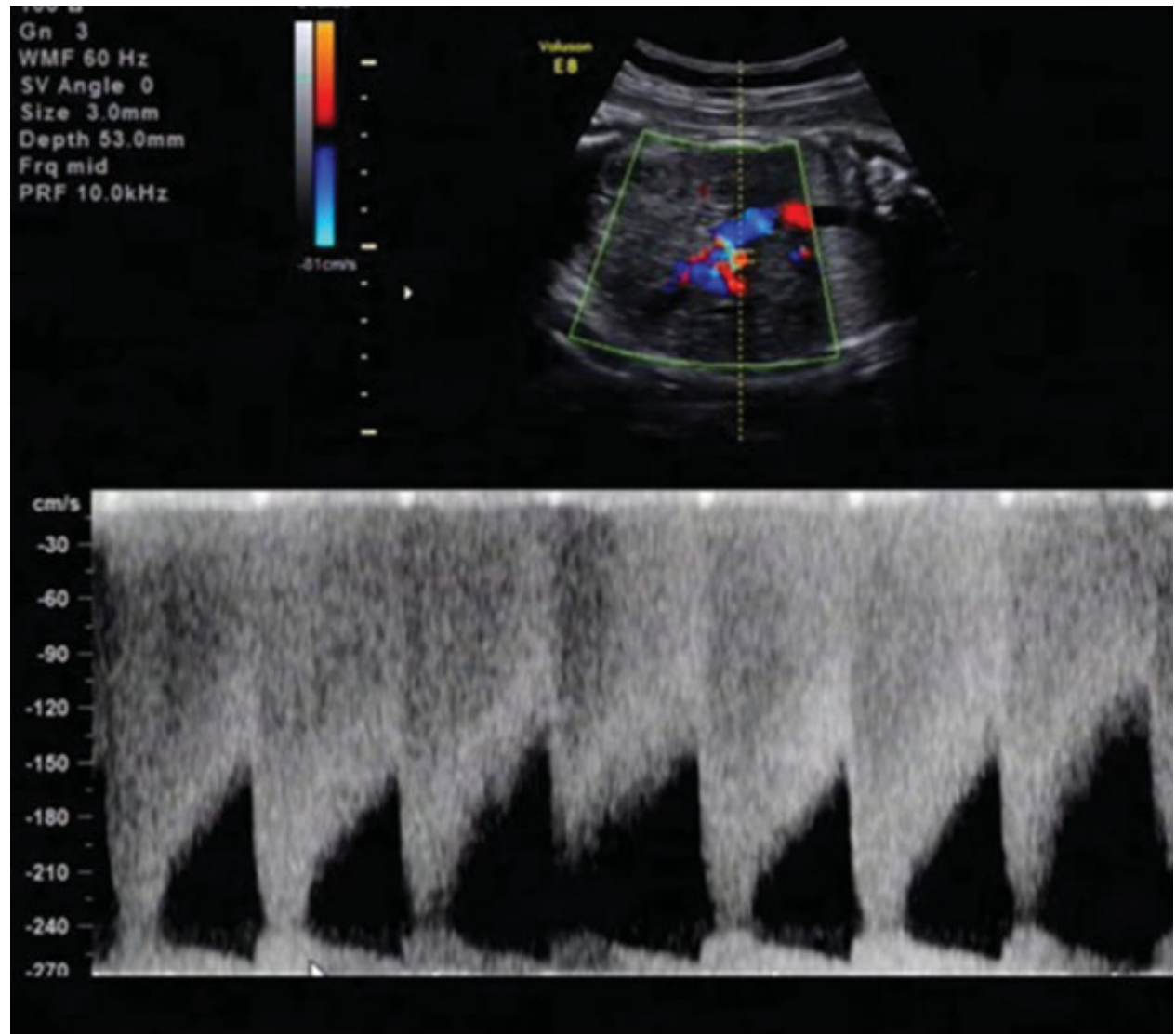

Fig. 1 Doppler revealed "saw-toothed" arterial flow pattern with extremely high-peak velocity $(240 \mathrm{~cm} / \mathrm{s})$ in the lesion.

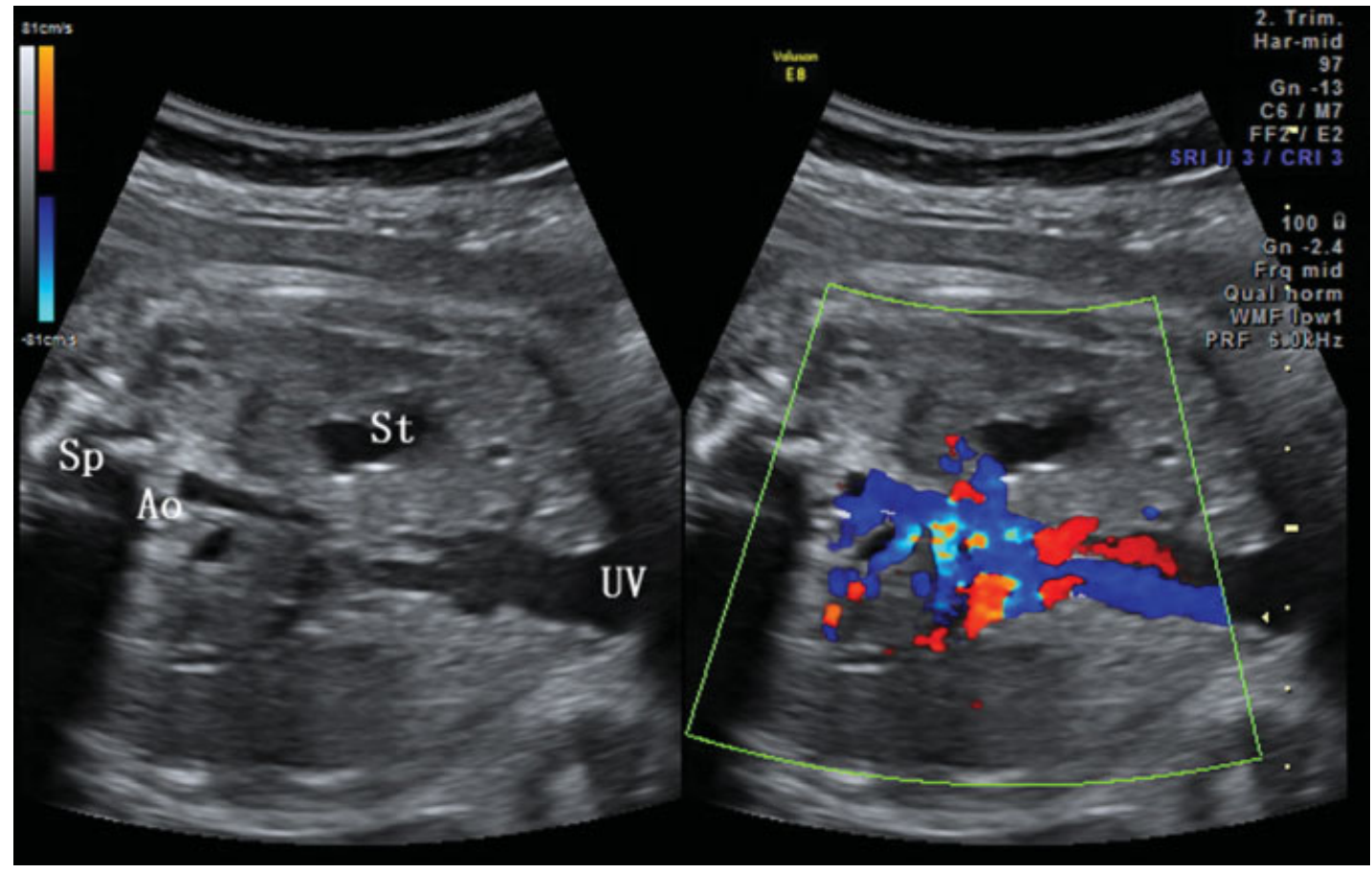

Fig. 2 Doppler imaging demonstrated a vascular structure in the middle part of the liver. The following vessels were in continuity with the lesion: an arterial vessel originating directly from the abdominal aorta, portal veins, and dilated umbilical vein. Ao, abdominal aorta; Sp, spine; St, stomach; UV, umbilical vein. 


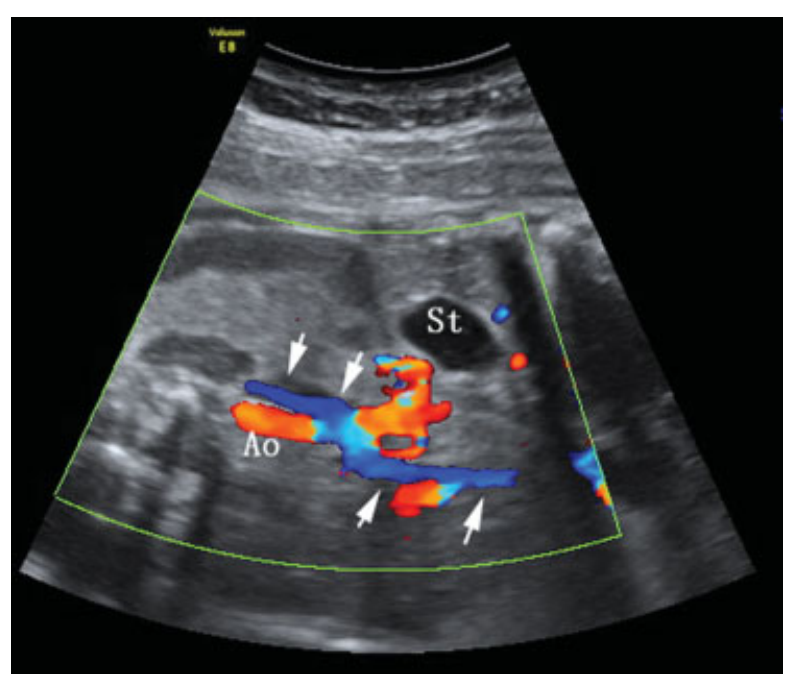

Fig. 3 The arrows demonstrate the course of IVC: the subrenal IVC ran upward along the left side of aorta, and then crossed the aorta anteriorly at the level of the kidney to join the normal IVC on the right. Ao, abdominal aorta; IVC, inferior vena cava; St, stomach.

literature found only one published report on the prenatal diagnosis of left-sided IVC in which the left-sided subrenal IVC continued as an azygos/hemiazygos vein with interrupted IVC. ${ }^{4}$ Left-sided IVC, although frequently asymptomatic, may result in Nutcracker syndrome postnatally due to left-sided IVC compression. ${ }^{5}$
Prenatal ultrasonography can facilitate the prenatal detection, provide close observation, and evaluate the complications and hemodynamic status of such lesions.

\section{Acknowledgment}

This study was supported by the National Clinical Key Subject (Medical Imageology) Construction Project of China (2013[544]).

\section{References}

1 Hartung J, Chaoui R, Kalache K, Tennstedt C, Bollmann R. Prenatal diagnosis of intrahepatic communications of the umbilical vein with atypical arteries (A-V fistulae) in two cases of trisomy 21 using color Doppler ultrasound. Ultrasound Obstet Gynecol 2000; 16(3):271-274

2 Tseng JJ, Chou MM, Lee YH, Ho ES. Prenatal diagnosis of intrahepatic arteriovenous shunts. Ultrasound Obstet Gynecol 2000; 15(5):441-444

3 Gedikbasi A, Oztarhan K, Sahin B, et al. Multidisciplinary approach to congenital multiple arterio-porto-caval malformation: case report. Am J Perinatol 2008;25(5):265-270

4 Vijayaraghavan SB, Raja V, Chitra TV. Interrupted inferior vena cava and left-sided subrenal inferior vena cava: prenatal diagnosis. J Ultrasound Med 2003;22(7):747-752

5 Yang BZ, Li Z, Wang ZG. Nutcracker syndrome due to left-sided inferior vena cava compression and treated with superior mesenteric artery transposition. J Vasc Surg 2012;56(3):816-818 